\title{
Current Evidence on Oral Antibiotics for Infective Endocarditis: A Narrative Review
}

\author{
Takaaki Kobayashi (D) - Tomo Ando · Judy Streit · Poorani Sekar
}

Received: July 19, 2019 / Published online: September 18, 2019

(C) The Author(s) 2019

\begin{abstract}
Infective endocarditis (IE) continues to be associated with high morbidity and mortality, even when treated with optimal antibiotic regimens. The selection of treatment depends on the causative pathogen, its antibiotic susceptibility profile, local and systemic complications and the presence of prosthetic materials or devices. Standard therapy typically involves 4-6 weeks of intravenous (IV) bactericidal therapy. However, there are instances in which IV antibiotic administration may be challenging due to cost, complications of IV access, adverse side-effects of the medication or concerns for misuse of the IV line. Current clinical guidance from the American Heart Association and the European Society of Cardiology cite scenarios where oral antibiotics can be considered for
\end{abstract}

Enhanced Digital Features To view enhanced digital features for this article go to https://doi.org/10.6084/ m9.figshare.9772064.

T. Kobayashi $(\bowtie) \cdot J$. Streit · P. Sekar

Division of Infectious Diseases, Department of Internal Medicine, University of Iowa Hospitals and Clinics, Iowa City, IA, USA

e-mail: taka.kobayashi1126@gmail.com

T. Ando

Division of Cardiology, Center for Interventional

Vascular Therapy, New York-Presbyterian Hospital/

Columbia University Medical Center, New York, NY, USA treatment of IE, though these situations are relatively infrequent and data to show their non-inferiority limited. Recently, a well-designed randomized clinical study reported favorable outcomes for partial oral antimicrobial therapy regimens given to patients with staphylococcal, streptococcal and enterococcal IE deemed clinically stable and without complications such as perivalvular abscess. Oral antibiotics, usually given in combination, were selected by infectious disease providers for their favorable pharmacologic properties and predicted bactericidal activity. There was a careful selection of patients who were transitioned to oral regimens. Before recommending routine use of oral antibiotics in the care of patients with IE, additional studies that better define eligible patients and that use regimens available in the countries that adopt this practice should be performed. If further studies confirm noninferior outcomes with partial oral antibiotics for the treatment of IE, medical treatment could be delivered in a simpler, more costeffective manner, and likely with lower rates of adverse side-effects.

Keywords: Infective endocarditis; IV therapy; Oral antibiotics; POET trial 


\section{INTRODUCTION}

Infective endocarditis involves the endocardial surface of the heart, most often the heart valves. Annual incidence of IE is 3-9 cases per 100,000 in industrialized countries [1]. Increased risk is conferred by the presence of prosthetic valves, implanted cardiac devices, unrepaired cyanotic heart disease, a history of endocarditis and intravenous drug use (IVDU). In-hospital mortality is known to be $20 \%$ worldwide in patients with IE [2] [3], while 5-year mortality was nearly $50 \%$ in a population-based study performed in California and New York [4].

The selection of optimal therapy (medical or medical plus surgical) and duration for IE depends on the pathogen, its antibiotic susceptibility profile, the presence of prosthetic material or implanted cardiac devices, and the presence of complications such as perivalvular abscess. Standard therapy usually involves 4-6 weeks of IV antimicrobials [5]. Such therapy may entail prolonged hospital stays, especially in circumstances where the delivery of outpatient IV antibiotic therapy is not deemed safe or feasible because of challenges with finances, patient transportation or line maintenance. In such challenging circumstances oral antibiotic therapy is an attractive alternative. However, data to support the use of oral antibiotics in the treatment of IE are limited. In this narrative review, we discuss the results of early studies on the efficacy of such therapy, pertinent information contained in current practice guidelines and recent data on the outcomes of patients with IE treated with partial oral therapy. This article is based on previously conducted studies and does not contain any studies with human participants or animals performed by any of the authors.

\section{ORAL ANTIBIOTICS FOR IE FROM THE AMERICAN HEART ASSOCIATION AND EUROPEAN SOCIETY OF CARDIOLOGY GUIDELINES}

Current guidelines from the American Heart Association (AHA) mention the option of oral antibiotics in several scenarios based on previously published case series and several trials [5]. For the therapy of uncomplicated right-sided methicillin-sensitive Staphylococcus aureus (MSSA) IE in febrile persons with IV drug use, a small prospective randomized non-blinded study by Heldman et al. compared the use of a predominantly 4-week oral antibiotic regimen (ciprofloxacin plus rifampin) to conventional treatment with IV oxacillin or vancomycin plus gentamicin [6]. Nineteen patients received only oral therapy vs. 25 who received IV therapy, and all completed 28 days of inpatient treatment. There were four treatment failures ( $5 \%$ in oral vs. $12 \%$ in IV) based on blood cultures performed 1 week after completion of therapy $(p=0.4)$. Drug toxicity was more common in the parenterally treated group ( $3 \%$ in oral vs. $62 \%$ in IV; $p<0.0001$ ). The authors concluded that for selected patients, oral ciprofloxacin plus rifampin was effective and associated with fewer adverse side effects than IV therapy. Dworkin et al. studied a similar oral regimen in right-sided IE [7]. In a non-comparative trial where study drugs were initiated after a mean of $34.4 \mathrm{~h}$ of conventional antibiotic for suspected IV drug-use related $S$. aureus IE (predominantly methicillinsusceptible), patients received approximately 1 week of IV ciprofloxacin which was then transitioned to high-dose oral ciprofloxacin for 3 weeks; oral rifampin was given throughout the whole treatment period. All ten patients who completed the study were cured based on resolution of symptoms and negative blood cultures at 4 weeks post therapy. Neither of these two studies led to widespread adoption of oral antibiotics for the treatment of staphylococcal endocarditis, in part due to very small sample sizes, non-comparative design for the latter and concern for decreasing susceptibility of $S$. aureus to ciprofloxacin over time.

Another organism in the AHA practice guidelines for which an oral antibiotic is discussed is Enterococcus faecium resistant to the usual first-line agents (ampicillin, vancomycin and aminoglycoside). In this challenging IE scenario, linezolid, an oxazolidinone antibiotic that is considered bacteriostatic but with activity against vancomycin-resistant Enterococcus (VRE), is a Class IIb recommendation (Level of Evidence C) [5]. In a small number of patients, 
linezolid was effective therapy for VRE IE [7]. Birmingham et al. reported cure in 17 of 22 cases treated with linezolid (77\%) for E. faecium IE [8]. The advantage of using linezolid is that it is highly bioavailable when taken by mouth. However, because of its inhibition of monoamine oxidase, concern for the development of serotonin syndrome when used concomitantly with serotonergic neuropsychiatric medications impedes its use. Other adverse side effects that warrant close monitoring include thrombocytopenia and anemia; less commonly, lactic acidosis, peripheral neuropathy, and ocular toxicity can occur [9]. However, treatment failures with linezolid for Vancomycin-resistant $E$. faecium and E. faecalis IE have been reported [10]. Subsequently, the superiority of daptomycin over linezolid was demonstrated in a retrospective study of 644 cases of bloodstream infections due to VRE (adjusted risk ratio 1.15 [1.02-1.30]; $p=0.026$ ) [11]. Therefore, linezolid is not considered first-line therapy for IE due to VRE, unless daptomycin non-susceptibility or intolerance is present.

Lastly, the AHA practice guidelines recommend the use of ciprofloxacin for native or prosthetic valve IE caused by the group of oral Gram-negative commensals referred to as the HACEK group (Haemophilus aphrophilus, Actinobacillus actinomycetemcomitans, Cardiobacterium hominis, Eikenella corrodens, Kingella kingae) (Class IIb recommendation, Level of Evidence C) [5]. The HACEK group is usually susceptible in vitro to fluoroquinolones [10]. A particular circumstance mentioned for their use is patient intolerance to ceftriaxone or other cephalosporin therapy. Very rarely, resistance to ciprofloxacin by members of the HACEK group has been described [12].

Practice guidelines of the European Society of Cardiology also cite the option of oral antibiotics in similar scenarios [13]. For rightsided Staphylococcus aureus IE in IV drug users, ciprofloxacin combined with rifampin is a secondary option, provided the strain is fully susceptible to both drugs, the case is uncomplicated and patient adherence is monitored carefully. For IE due to $S$. aureus, sulfamethoxazole/trimethoprim (IV for first week followed by oral for 5 weeks) and oral clindamycin are listed as alternative options (Class IIb recommendation, Level of Evidence C). For IE due to HACEK organisms, the European guidelines mention fluoroquinolones as a less well-validated alternative.

\section{ADDITIONAL LITERATURE EVALUATING THE EFFICACY OF ORAL THERAPY FOR IE}

To identify additional pertinent studies reporting the use of oral antibiotics for the treatment of IE, PubMed, Embase and Google Scholar were searched through April 2019. The PubMed search was as follows: $\left(\left(()\left(\left({ }^{\prime \prime}\right.\right.\right.\right.$ endocarditis"[MeSH Terms] OR "endocarditis"[All Fields]) AND ((( "anti bacterial agents"[Pharmacological Action] OR "anti-bacterial agents" [MeSH Terms] OR antibiotics[tw]) OR (anti bacterial agents[tw])) OR (antibacterial agents[tw])) OR (anti biotics[tw]))) AND ("administration, oral"[MeSH Terms] OR "oral"[All Fields])) NOT (dental[tw] OR dentist[tw] OR dentists[tw]))))) NOT ((animals[mh] NOT (humans[mh] AND animals[mh]))). This strategy was translated for Embase. PubMed and Embase searches limited to the last 10 years yielded 127 and 154 records, respectively including duplicates. Google Scholar identified an additional 22 records. For our narrative review we included all systematic reviews and prospective studies and excluded retrospective studies with study populations less than 30 patients, non-English and animal studies. We reviewed all titles and abstracts and narrowed the list to four relevant studies in the past 10 years. We found one systematic review published on the subject in 2014; we thoroughly reviewed its reference list for additional pertinent studies. This provided us with six relevant studies older than 10 years. These combined ten studies were included in this narrative review and are summarized in Table 1.

Schein et al. retrospectively studied, before widespread availability of penicillin, 81 patients with predominantly streptococcal endocarditis treated with oral sulfonamides [14]. The duration of therapy was widely variable (10 days to 14 weeks). The cure rate was a dismal $10 \%$ when evaluated at several years' follow-up. 


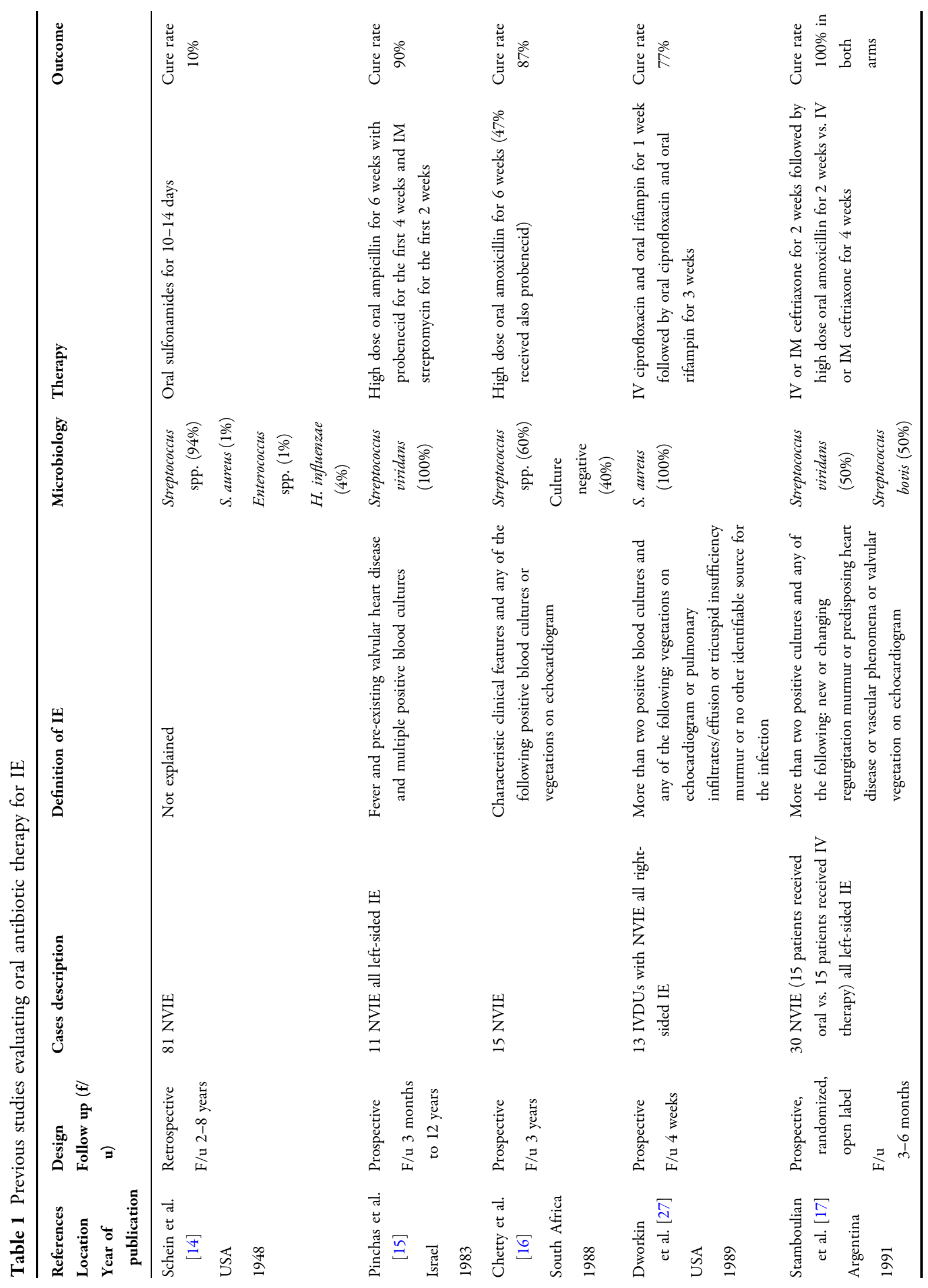




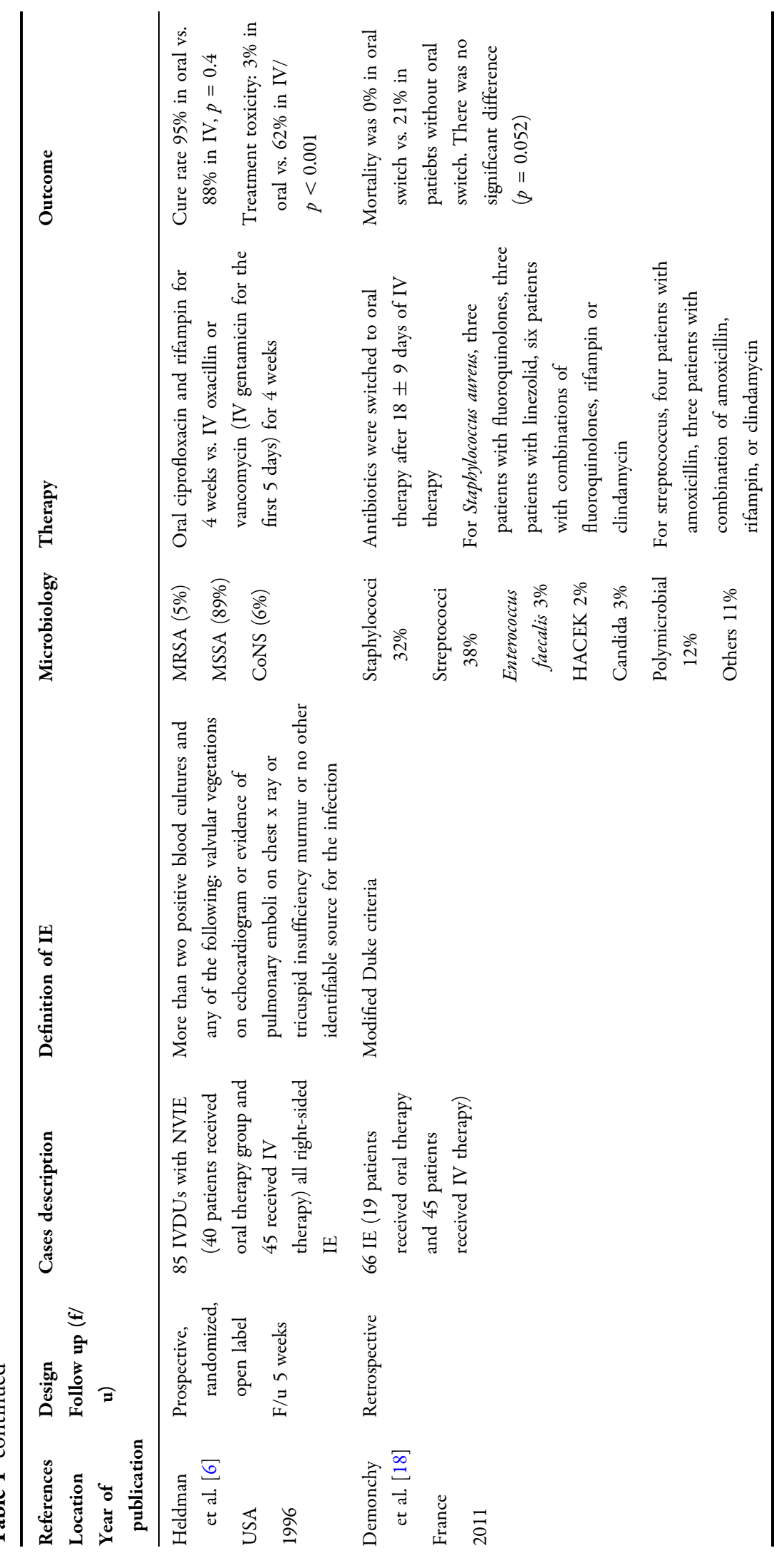




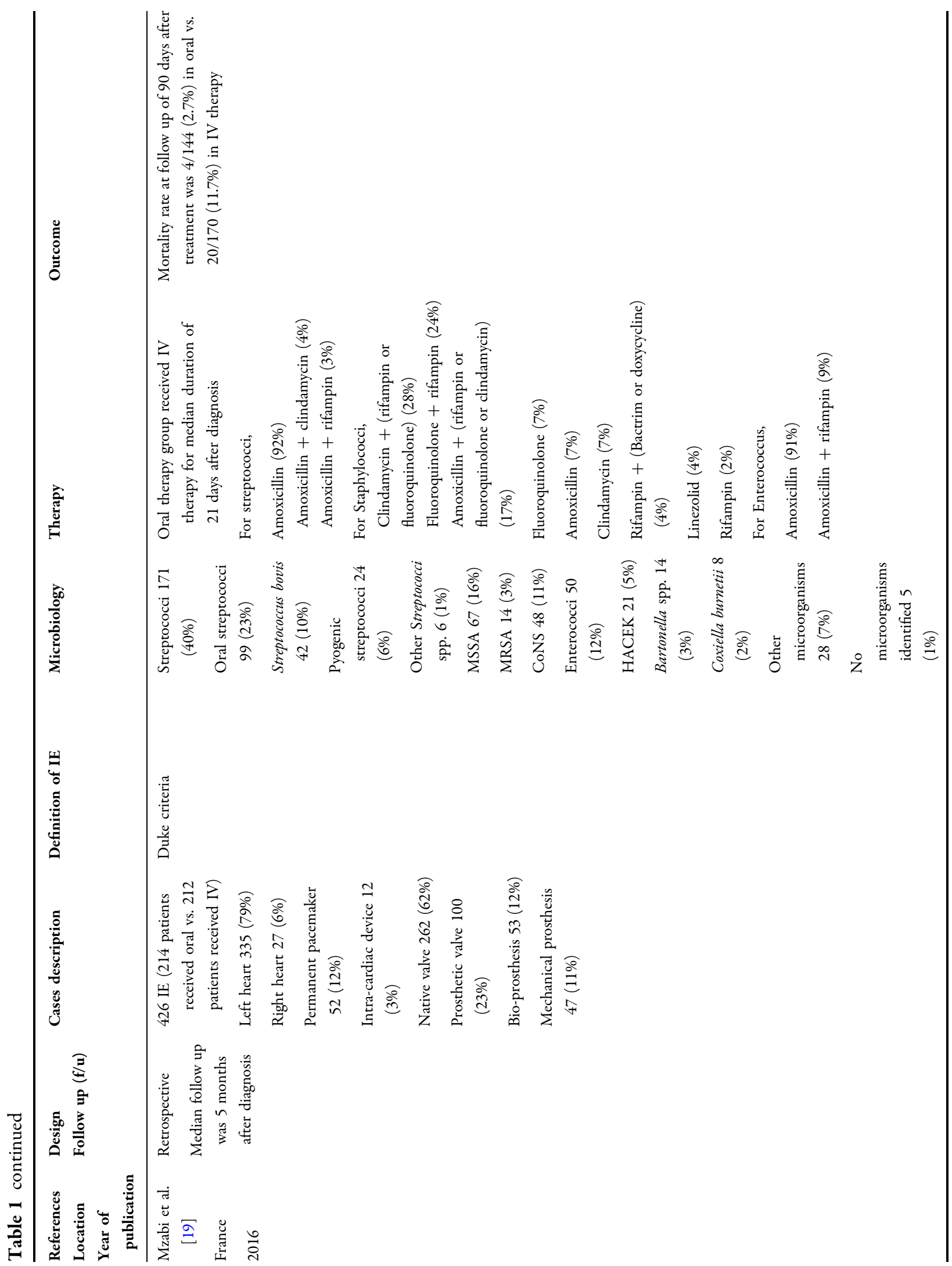




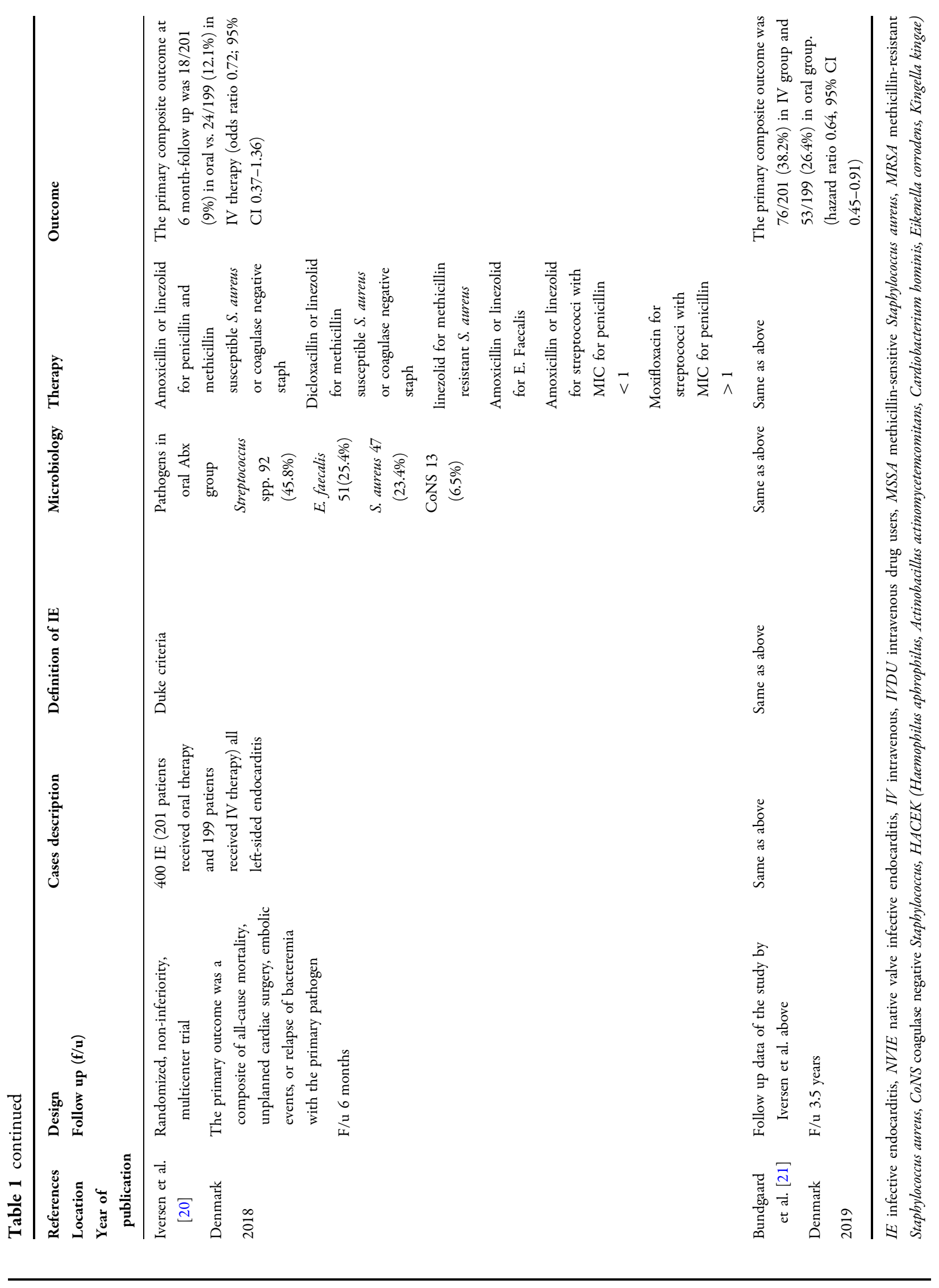


A few decades later, Pinchas et al. [15] reported outcomes of 11 patients with uncomplicated Streptococcus viridans endocarditis who received 6 weeks of oral ampicillin with 2 weeks of concurrent intra-muscular streptomycin and 4 weeks of probenecid. All blood cultures taken after treatment were negative and they remained free from recurrences during followup at 3 months to 12 years.

Progressing along the chronology of publications included in this report, a prospective study by Chetty et al. assessed outcomes of 15 patients with uncomplicated ampicillin-susceptible Streptococcus IE treated with 6 weeks of high-dose oral amoxicillin [16]. Twelve (80\%) patients responded to treatment and remained well at 3 years. There were three deaths; one at day 7 due to sudden aortic cusp rupture, and two later deaths due to pulmonary and cerebral embolism. One patient relapsed 8 weeks after oral therapy and responded to conventional IV treatment.

In the late 1980s, Stamboulian et al. conducted a prospective, randomized study of 30 patients with penicillin-susceptible streptococcal IE [17] to assess partial oral therapy. Fifteen patients received ceftriaxone for 4 weeks while the other 15 received the same dose of ceftriaxone for 2 weeks followed by oral amoxicillin for 2 weeks. Clinical cure was achieved in all patients in both groups. Broad exclusion criteria were applied, including the presence of heart failure, severe aortic insufficiency, conduction system abnormalities, recurrent thromboembolic disease and prosthetic valve endocarditis.

Moving into the new millennium, Demonchy et al. [18] conducted a retrospective study of adult patients with endocarditis hospitalized between 2007 and 2009. In a total of 66 patients, 19 (29\%) patients received an oral antibiotic after $18 \pm 9$ days of IV antibiotics. A switch to oral antibiotic therapy was not associated with increased risk of mortality compared to those who had only IV therapy $(0 \%$ mortality in oral switch vs. $21 \%$ without, $p=0.052$ ).

A significantly larger retrospective study with 426 patients treated for IE between 2000 and 2012 was published by Mzabi et al. [19]. Treatment followed the European IE guidelines except that switching to oral antibiotics was allowed after 7 days of IV therapy if the patient's general condition had improved. In all, 214 patients fulfilled these criteria after a median of 21 days on IV therapy. These patients had fewer comorbidities, less-severe disease, and were less likely to be infected with $S$. aureus than those who remained on IV therapy. Among patients transitioned to oral therapy (median follow-up, 9 months), two relapses, four reinfections, and 16 deaths occurred; among those who continued on IV antibiotics, nine relapses, eight reinfections, and 76 deaths occurred (median follow-up, 3 months). A multivariate analysis that controlled for age, sex, pathogen type and comorbidities indicated that switching to oral treatment was not associated with excess risk for relapse or reinfection. This study suggested that the use of oral antibiotics for a portion of treatment of IE was non-inferior to a full course of IV therapy for some patients.

A recent study that has generated much interest regarding the benefits of relatively early transition to oral antibiotics is the Partial Oral vs. Intravenous Antibiotic Treatment (POET) study conducted by Iversen et al. [20]. This was a randomized, prospective, multicenter noninferiority trial conducted in Denmark between 2011 and 2017. From 1954 patients screened because of suspected IE, 400 patients (20\%) with left-sided endocarditis fulfilled modified Duke criteria and were randomly assigned to IV or oral therapy after an initial 10 days of IV therapy. Common reasons for exclusion were inability to meet the modified Duke criteria (28\%), lack of consent (19\%), and infection with bacteria not included in the study protocol (11\%). The primary endpoint was a composite of all-cause mortality, relapse of bacteremia after 6 months, embolic events, and other complications. The two groups were well balanced with regard to baseline characteristics. In patients treated with partial oral therapy, the causative organisms were Streptococcus spp. $(45.8 \%)$, Enterococcus faecalis $(25.4 \%), S$. aureus $(23.4 \%)$ and coagulase negative staphylococci (6.5\%). Methicillin-resistant $S$. aureus was rare. All patients were followed for 6 months. The composite outcome occurred in $12.5 \%$ of the IV group versus $9 \%$ of the oral therapy group (odds ratio $0.72 ; 95 \%$ confidence interval $0.37-1.36$ ). 
Adverse effects from antibiotics were reported in $12(6 \%)$ patients in the IV treatment group and $10(5 \%)$ patients in the oral therapy group $(p=0.66)$. The results suggested that an oral antibiotic switch is an option for the management of stable patients with IE. In addition, Bundgaard et al. [21] performed a post hoc longterm follow-up review of these patients. After a median follow up of 3.5 years, the primary composite outcome had occurred in 76 patients in the intravenously treated group (38.2\%) and in 53 patients in the orally treated group (26.4\%) (hazard ratio $0.64,95 \%$ confidence interval 0.45-0.91).

Al-Omari et al. [22] conducted a systematic review on oral antibiotic therapy for endocarditis in 2014 before Mzabi's study and the POET study were published. Their main finding was that cure rates for endocarditis caused by susceptible organisms treated with well-matched oral antibiotic regimens ranged between 75 and $100 \%$. However, they noted that a majority of the studies included in their analysis had poor methodological quality and broad heterogeneity in study populations and study design. These factors prevented them from calculating any meaningful pooled estimates.

\section{DISCUSSION}

Current practice guidelines for IE recommend IV therapy for most episodes. However, recent studies have shown IV antibiotics might not be necessary for the entirety of the treatment course, depending on clinical circumstances of the case. There are multiple risks and high costs associated with extended courses of intravenous antibiotics, including procedural complications from intravenous lines, central-line associated infections, and thrombosis [23]. Outpatient parenteral antibiotic therapy (OPAT) allows patients to receive parenteral antimicrobials outside of acute care hospitals. Internationally, OPAT utilization has considerably increased, since its introduction in the 1970s, to treat a variety of infections [24]. It is estimated that more than 250,000 patients receive OPAT annually, and its use is growing by more than $10 \%$ annually in the United States [25]. Previous studies have demonstrated OPAT-related adverse events occur in about $25 \%$ of patients and most commonly involve antibiotic reactions, hematologic or gastrointestinal adverse effects, or IV access complications [26]. Oral antimicrobial therapy for endocarditis would offer several potential benefits including lower costs, fewer side effects and greater ease for patients.

The study by Mzabi et al. [19] indicated noninferior outcomes for patients transitioned to oral antibiotics when good clinical response had been achieved while on IV therapy, but the retrospective nature and broad range of days before transition to oral therapy makes application of the data a challenge. The POET study [20], a well-designed prospective randomized trial of patients with Gram-positive left-sided IE provides stronger evidence that in select patients, carefully chosen oral antimicrobial regimens produce non-inferior outcomes compared to all-IV therapy. There was intensive clinical assessment of the patients, including a TEE shortly before transition to oral therapy, Infectious Disease expertise rendered for the selection of oral regimens, and very close outpatient follow-up. Of note, MRSA was not significantly represented in the oral group and few patients had IVDU as a risk factor for IE. Therefore, the findings may not reflect the patient profiles in other countries, including the US. The fact that only $20 \%$ of the screened population was randomized is one reason that widespread adoption of partial oral antibiotic therapy is premature until further studies validate the findings and confirm which features of patients with IE allow for the safe transition to oral treatment. On the basis of these recent data, targeted oral therapy likely has a role in the treatment of selected patients with leftsided IE, but more information is needed to recommend its widespread adoption.

\section{CONCLUSIONS}

There is increasing evidence that in some patients with IE, transition to oral antibiotics after achieving a clinical response with IV therapy may have good efficacy and reduced adverse side-effects when compared to all-IV 
therapy. If such positive outcomes are confirmed in further studies with good delineation regarding patient and oral regimen options, the benefits would include significantly lower costs to the healthcare system, reduced risks of adverse side-effects associated with prolonged intravenous access, and ease for the patient.

\section{ACKNOWLEDGEMENTS}

We thank Heather Healy for her help with the literature search.

Funding. No funding or sponsorship was received for this study or publication of this article.

Authorship. All names authors meet the International Committee of Medical Journal Editors (ICJME) criteria for authorship of this article, take responsibility for the integrity of the work as a whole, and have given their approval for this version to be published.

Disclosures. Takaaki Kobayashi, Tomo Ando, Judy Streit and Poorani Sekar have nothing to disclose.

Compliance with Ethics Guidelines. This article is based on previously conducted studies and does not contain any studies with human participants or animals performed by any of the authors.

Data Availability. Data sharing is not applicable to this article as no datasets were generated or analyzed during the current study.

Open Access. This article is distributed under the terms of the Creative Commons Attribution-NonCommercial 4.0 International License (http://creativecommons.org/licenses/ by-nc/4.0/), which permits any noncommercial use, distribution, and reproduction in any medium, provided you give appropriate credit to the original author(s) and the source, provide a link to the Creative Commons license, and indicate if changes were made.

\section{REFERENCES}

1. Hoen B, Duval X. Clinical practice. Infective endocarditis. N Engl J Med. 2013;368(15):1425-33.

2. Murdoch DR, Corey GR, Hoen B, Miro JM, Fowler VG Jr, Bayer AS, Karchmer AW, Olaison L, Pappas PA, Moreillon P, et al. Clinical presentation, etiology, and outcome of infective endocarditis in the 21st century: the International Collaboration on Endocarditis-Prospective Cohort Study. Arch Intern Med. 2009;169(5):463-73.

3. Federspiel JJ, Stearns SC, Peppercorn AF, Chu VH, Fowler VG Jr. Increasing US rates of endocarditis with Staphylococcus aureus: 1999-2008. Arch Intern Med. 2012;172(4):363-5.

4. Toyoda N, Chikwe J, Itagaki S, Gelijns AC, Adams $\mathrm{DH}$, Egorova NN. Trends in infective endocarditis in California and New York State, 1998-2013. JAMA. 2017;317(16):1652-60.

5. Baddour LM, Wilson WR, Bayer AS, Fowler VG Jr, Tleyjeh IM, Rybak MJ, Barsic B, Lockhart PB, Gewitz $\mathrm{MH}$, Levison $\mathrm{ME}$, et al. Infective endocarditis in adults: diagnosis, antimicrobial therapy, and management of complications: a scientific statement for healthcare professionals from the American Heart Association. Circulation. 2015;132(15):1435-86.

6. Heldman AW, Hartert TV, Ray SC, Daoud EG, Kowalski TE, Pompili VJ, Sisson SD, Tidmore WC, vom Eigen KA, Goodman SN, et al. Oral antibiotic treatment of right-sided staphylococcal endocarditis in injection drug users: prospective randomized comparison with parenteral therapy. Am J Med. 1996;101(1):68-76.

7. Falagas ME, Manta KG, Ntziora F, Vardakas KZ. Linezolid for the treatment of patients with endocarditis: a systematic review of the published evidence. J Antimicrob Chemother. 2006;58(2):273-80.

8. Birmingham MC, Rayner CR, Meagher AK, Flavin SM, Batts DH, Schentag JJ. Linezolid for the treatment of multidrug-resistant, gram-positive infections: experience from a compassionate-use program. Clin Infect Dis. 2003;36(2):159-68.

9. Lawrence KR, Adra M, Gillman PK. Serotonin toxicity associated with the use of linezolid: a review of postmarketing data. Clin Infect Dis. 2006;42(11):1578-83.

10. Tsigrelis C, Singh KV, Coutinho TD, Murray BE, Baddour LM. Vancomycin-resistant Enterococcus faecalis endocarditis: linezolid failure and strain characterization of virulence factors. J Clin Microbiol. 2007;45(2):631-5. 
11. Britt NS, Potter EM, Patel N, Steed ME. Comparison of the effectiveness and safety of linezolid and daptomycin in vancomycin-resistant enterococcal bloodstream infection: a National Cohort Study of Veterans Affairs Patients. Clin Infect Dis. 2015;61(6):871-8.

12. Coburn B, Toye B, Rawte P, Jamieson FB, Farrell DJ, Patel SN. Antimicrobial susceptibilities of clinical isolates of HACEK organisms. Antimicrob Agents Chemother. 2013;57(4):1989-91.

13. Habib G, Lancellotti P, Antunes MJ, Bongiorni MG, Casalta JP, Del Zotti F, Dulgheru R, El Khoury G, Erba PA, Iung B, et al. The 2015 ESC guidelines for the management of infective endocarditis: the task force for the management of infective endocarditis of the European Society of Cardiology (ESC) Endorsed by: European Association for CardioThoracic Surgery (EACTS), the European Association of Nuclear Medicine (EANM). Eur Heart J. 2015;36(44):3075-128.

14. Schein J, Baehr G. Sulfonamide therapy of subacute bacterial endocarditis. Am J Med. 1948;4(1):66-72.

15. Pinchas A, Lessing J, Siegman-Igra Y, Liron M. Oral treatment of bacterial endocarditis. Isr J Med Sci. 1983;19(7):646-8.

16. Chetty S, Mitha AS. High-dose oral amoxycillin in the treatment of infective endocarditis. S Afr Med J. 1988;73(12):709-10.

17. Stamboulian D, Bonvehi P, Arevalo C, Bologna R, Cassetti I, Scilingo V, Efron E. Antibiotic management of outpatients with endocarditis due to penicillin-susceptible streptococci. Rev Infect Dis. 1991;13(Suppl 2):S160-3.

18. Demonchy E, Dellamonica P, Roger PM, Bernard E, Cua E, Pulcini C. Audit of antibiotic therapy used in 66 cases of endocarditis. Med Mal Infect. 2011;41(11):602-7.

19. Mzabi A, Kerneis S, Richaud C, Podglajen I, Fernandez-Gerlinger MP, Mainardi JL. Switch to oral antibiotics in the treatment of infective endocarditis is not associated with increased risk of mortality in non-severely ill patients. Clin Microbiol Infect. 2016;22(7):607-12.

20. Iversen K, Ihlemann N, Gill SU, Madsen T, Elming H, Jensen KT, Bruun NE, Hofsten DE, Fursted K, Christensen JJ, et al. Partial oral versus intravenous antibiotic treatment of endocarditis. N Engl J Med. 2019;380(5):415-24.

21. Bundgaard $H$, Ihlemann N, Gill SU, Bruun NE, Elming H, Madsen T, Jensen KT, Fursted K, Christensen JJ, Schultz M, et al. Long-term outcomes of partial oral treatment of endocarditis. N Engl J Med. 2019;380(14):1373-4.

22. Al-Omari A, Cameron DW, Lee C, Corrales-Medina VF. Oral antibiotic therapy for the treatment of infective endocarditis: a systematic review. BMC Infect Dis. 2014;14:140.

23. Hale AJ, Snyder GM, Ahern JW, Eliopoulos G, Ricotta D, Alston WK. When are oral antibiotics a safe and effective choice for bacterial bloodstream infections? An evidence-based narrative review. J Hosp Med. 2018;13(5):328-35.

24. Gesser RM, McCarroll KA, Woods GL. Evaluation of outpatient treatment with ertapenem in a double blind controlled clinical trial of complicated skin/ skin structure infections. J Infect. 2004;48(1):32-8.

25. Suleyman G, Kenney R, Zervos MJ, Weinmann A. Safety and efficacy of outpatient parenteral antibiotic therapy in an academic infectious disease clinic. J Clin Pharm Ther. 2017;42(1):39-43.

26. Chapman AL, Seaton RA, Cooper MA, Hedderwick S, Goodall V, Reed C, Sanderson F, Nathwani D. Group BBOPGPRW: good practice recommendations for outpatient parenteral antimicrobial therapy (OPAT) in adults in the UK: a consensus statement. J Antimicrob Chemother. 2012;67(5):1053-62.

27. Dworkin RJ, Lee BL, Sande MA, Chambers HF. Treatment of right-sided Staphylococcus aureus endocarditis in intravenous drug users with ciprofloxacin and rifampicin. Lancet. 1989;2(8671):1071-3. 